Revista Española de Antropología Americana ISSN: 0556-6533

http://dx.doi.org/10.5209/reaa.71750

\title{
Tecnología, cultura material y materialidad: aproximaciones conceptuales a las actividades del ser humano y sus producciones materiales
}

\author{
Carlos G. Landa ${ }^{1}$ y Nicolás C. Ciarlo ${ }^{2}$
}

Recibido: 20 de diciembre de 2018 / Aceptado: 21 de febrero 2020

Resumen. Los estudios sobre el quehacer humano y sus evidencias materiales han ocupado un espacio de relevancia en la agenda de varias disciplinas sociales. Estas investigaciones revisten sumo interés para el conocimiento de diferentes aspectos de la vida pasada y presente de las personas. En conjunto, abarcan diferentes contextos temporales y espaciales, aproximaciones teóricas, problemáticas, materiales, etc. En este trabajo se realiza una revisión de las principales conceptualizaciones utilizadas en Antropología, Arqueología y, de modo complementario, Sociología e Historia, desde finales del siglo XIX. Puntualmente, se pasa revista a las nociones de cultura material, materialidad, tecnología y conceptos afines, teniendo en cuenta sus semejanzas y diferencias. Se discuten algunas cuestiones del proceso de conceptualización, la especificidad de cada disciplina y la dinámica de comunicación entre ellas, a partir de lo cual se vislumbra el enorme potencial que supone un acercamiento interdisciplinar. Palabras clave: tecnología; cultura material; materialidad; análisis conceptual; teoría en Ciencias Sociales.

\section{[en] Technology, Material Culture, and Materiality: Conceptual Approaches to the Human Being Activities and their Material Productions}

\begin{abstract}
Researches on human activities and their material evidence have occupied an important place in the agenda of many social disciplines. These studies are of an outstanding interest to the understanding of various aspects of people' past and current life. As a whole, cover different temporal and spatial contexts, theoretical approaches, subjects, materials, etc. This paper presents a review of the main conceptualizations used in Anthropology, Archeology and, complementarily, Sociology and History, since the late 19th century. In particular, a theoretical revision of the notions of material culture, materiality, technology and related concepts, taking into account their similarities and differences, is exposed. Some issues of the process of conceptualization, the specificity of each discipline and the dynamics of communication between them are discussed, scenario from which the high potential that an interdisciplinary approach suppose is sighted.
\end{abstract}

Keywords: technology; material culture; materiality; conceptual analysis; theory in social sciences.

Sumario: 1. Introducción a la materia. 2. Términos, conceptos y teorías: algunas consideraciones generales. 3. Tecnología. 4. Cultura material. 5. Materialidad. 6. Consideraciones finales. 7. Referencias.

\footnotetext{
1 Consejo Nacional de Investigaciones Científicas y Técnicas (CONICET), Instituto de Arqueología de la Facultad de Filosofía y Letras de la Universidad de Buenos Aires. carlosglanda@gmail.com.

2 Consejo Nacional de Investigaciones Científicas y Técnicas (CONICET), Instituto de Arqueología de la Facultad de Filosofía y Letras de la Universidad de Buenos Aires. nciarlo@conicet.gov.ar.
} 
Cómo citar: Landa, Carlos G. y Nicolás C. Ciarlo. 2020. "Tecnología, cultura material y materialidad: aproximaciones conceptuales a las actividades del ser humano y sus producciones materiales". Revista Española de Antropología Americana 50: 191-210.

\section{Introducción a la materia}

Los materiales utilizados por el ser humano, desde los primeros cantos rodados sin modificar previamente a su uso -implementados por los homínidos para romper los huesos de las carcasas animales y acceder a la médula- hasta las máquinas más grandes y complejas jamás construidas (e.g. el transbordador espacial Saturno V, la embarcación Freedom of the Seas, el acelerador y colisionador de partículas Large Hadron Collider o "Máquina de Dios", por citar algunos de los más reconocidos), pueden ser denominados conjuntamente como artefactos u objetos ${ }^{3}$, aunque muchos de ellos sean verdaderas estructuras con cientos de miles de partes componentes. Estos artefactos forman parte de un complejo sistema dentro de cada sociedad, por lo que no constituyen una entidad aislada, con un lógica y mecanismo de funcionamiento propios. Cualquiera sea la denominación que se le otorga al conjunto, como veremos, implícita o explícitamente suele asumirse como premisa general que el mismo está constituido por materiales, personas, conocimientos, espacios, etc., íntimamente relacionados entre $\mathrm{si}^{4}$.

Las investigaciones teóricas en torno a la transformación material de la naturaleza por el ser humano y su vinculación con diversos aspectos socio-culturales, han sido desarrolladas por varias disciplinas y especialidades dentro de las Ciencias Sociales, fundamentalmente la Antropología, Arqueología, Sociología e Historia. El panorama general está regado de discusiones sobre una diversidad de aspectos, tales como la definición del tema, las relaciones entre lo material y las personas en el pasado y en la actualidad -desde el individuo hasta, podría decirse, el ser humano como especie-, las metodologías de análisis, etc., aunque por lo general no trascendieron las fronteras disciplinares. Por otro lado, pueden apreciarse orientaciones semejantes -desarrolladas aparentemente de forma independiente y, por lo general, no simultánea- con relación a algunas de las aproximaciones en cuestión. Sin embargo, puede reconocerse que en cada ámbito se mantiene una terminología específica (e.g. tecnología, cultura material y materialidad). Lo anterior supone ciertos inconvenientes teóricos y prácticos con relación al uso de los términos, en especial a nivel comparativo, teniendo en cuenta que cada uno suele poseer múltiples acepciones -en el mejor de los casos, un sentido amplio y otro/s más restringido/s, según se considere la disciplina y/o el marco teórico de cada investigador-.

Este trabajo presenta un estado de la cuestión de la terminología utilizada en Ciencias Sociales, en particular Antropología, Arqueología y, complementariamen-

\footnotetext{
Teniendo en cuenta que muchas otras especies animales utilizan o fabrican artefactos -tal como fueron definidos-, estos términos podrían acompañarse de algún calificativo más específico (e.g. objetos culturales). Aquí simplemente se hará mención sencilla de los términos, siempre con referencia exclusiva a las producciones del ser humano.

4 Es importante tener en cuenta que el modo en que los artefactos son nombrados y clasificados responde a una operación subjetiva realizada por un observador con miras a dar respuesta a ciertas preguntas (Potter 1992: 19).
} 
te, Sociología e Historia, para representar (conceptualmente) en algún modo -ya se verá en cada caso- al quehacer humano y sus producciones materiales, usualmente a una escala no menor que la de una sociedad, cultura o grupo determinado. Más específicamente, se centra en el análisis sincrónico y diacrónico - desde fines del siglo XIX hasta el presente- de los siguientes conceptos: tecnología, cultura material y materialidad. El marco temporal escogido abarca de modo aproximado desde el momento de constitución formal de las disciplinas hasta perspectivas recientes, si bien el escrito no pretende ser un compendio exhaustivo sobre la multiplicidad de miradas que han surgido a lo largo de los años ${ }^{5}$. De igual modo, la discusión se fundamenta en obras ya clásicas dentro del campo de las referidas disciplinas, así como en trabajos de síntesis sobre conceptos específicos, que en su conjunto han sido desarrollados principalmente por autores europeos y americanos. Sin ánimos de enfatizar una perspectiva en concreto, se examinará qué aspectos del mundo material (y no material) de las sociedades pasadas y presentes son subsumidos por determinados conceptos, de importancia tanto teórica como práctica; qué términos, simples o complejos, han sido utilizados para comunicar estos últimos y por qué; así como las semejanzas y diferencias significativas (de contenido conceptual o intensión) al interior y entre las disciplinas mencionadas desde el tiempo de su constitución formal. Se discutirán algunas cuestiones relacionadas con el proceso de conceptualización, la especificidad de cada disciplina y la dinámica interdisciplinar. Concretamente, el foco está puesto en el análisis descriptivo e interpretativo de la terminología y los conceptos específicos (a nivel semántico y pragmático), desde la óptica de cada disciplina. A lo largo del escrito se abordan también cuestiones epistemológicas estructurales y dinámicas (Gianella 1995: 21), de interés para comprender el significado de los términos utilizados, la vinculación de las teorías y sus referentes (las personas y las cosas -no individualizadas-, y las relaciones entre ellas) y las innovaciones conceptuales más relevantes. Asimismo, las reflexiones esbozadas pueden tener implicaciones para un análisis metateórico, o investigación de segundo orden (Díez y Moulines 1999: 17).

Antes de enfocarnos en el tema central del trabajo, realizaremos algunas consideraciones epistemológicas generales que permitirán comprender de modo más cabal la presentación sobre los conceptos de interés.

\section{Términos, conceptos y teorías: algunas consideraciones generales}

En el ámbito de la ciencia, suele hablarse de disciplinas para hacer referencia a cada uno de sus campos constituyentes, que están dedicados a conocer -comprender, por intermedio de la aplicación de ciertos procedimientos, i.e. métodos científicos, que permiten realizar a la vez que justificar afirmaciones acerca de- ciertos sectores de la realidad, natural y social. En el caso de las ciencias fácticas (o empíricas), dentro de las cuales se ubican las Ciencias Sociales, la labor científica consiste en analizar

\footnotetext{
Si bien el interés por las diversas manifestaciones materiales del ser humano (las cosas) puede remontarse a muchos siglos atrás, su estudio en el marco de las disciplinas sociales comenzó a desarrollarse a la par de la constitución de las mismas desde la segunda mitad del siglo XIX en los países industriales de Europa occidental. Muchos de los trabajos pioneros estuvieron influidos fuertemente por una concepción positivista del mundo social, en la que los artefactos y demás manifestaciones culturales fueron considerados como evidencias diagnósticas de ciertas etapas del progreso del ser humano.
} 
y ofrecer respuesta -mediante teorización- a problemas relativos a las entidades que conforman determinado dominio (ver Klimovsky 2005: 21-25). El lenguaje es primordial, dado que constituye el medio por el cual se conceptualiza la realidad analizada, es decir el canal de comunicación empleado por la ciencia. Para referirse a las cosas o entidades estudiadas, los científicos utilizan un vocabulario particular, i.e. una terminología.

La terminología constituye un conjunto o sistema de términos. A diferencia de la nomenclatura, cuyos elementos denominan (por extensión, a modo de "etiquetas") los objetos de la realidad a los que se aplican: "las unidades de una terminología designan -y denominan- nociones que pueden ser interpretables" (Torres 1994: 85).

Muchos de los términos utilizados en el ámbito científico provienen del lenguaje ordinario (común, vulgar), aunque las entidades a las que refieren no necesariamente se corresponden, es decir que pueden diferir sus designaciones. Otros conceptos, en cambio, son acuñados en el marco de ciertas teorías, pudiéndose o no usar palabras nuevas (ver Klimovsky 2005: 55-63). Esta terminología específica se construye conforme a determinada estructuración e interpretación (científica) de la realidad (Coseriu 1977: 96). Así, mientras aquella pertenece al metalenguaje de una lengua, sus unidades integran el metaléxico (Torres 1994: 86-87).

La pluralidad terminológica presente en la literatura científica refiere muchas veces a cosas o nociones semejantes; en algunos casos existe cierto solapamiento respecto de los conceptos involucrados, mientras que en otros la distinción entre estos es menos ambigua. Por otro lado, debido a la multiplicidad de perspectivas teóricas, a los diferentes "juegos de lenguaje" (sensu Wittgenstein 2006) podría decirse, un mismo término suele englobar-muchas veces de forma implícita-diversos campos designativos. Esto se aprecia, en sincronía, especialmente en aquellas disciplinas en las que el "pensamiento divergente" (Kuhn 1977, entre otros) es materia habitual. Las variables involucradas en la producción de nuevas ideas al interior de una comunidad académica son diversas. Desde una perspectiva de la sociología de la ciencia, Collins (1989) destacó que los efectos de las condiciones (externas) políticas y económicas, entre otras, aunque son relevantes, se ven filtrados a través de la estructuración interna de los grupos intelectuales, en particular por el proceso competitivo de apropiación de ideas entre sus integrantes.

Las preguntas acerca de qué son los conceptos (i.e. cuál es su naturaleza) y qué rol cumplen han motivado extensas reflexiones y discusiones filosóficas a lo largo de los siglos.

Diversas escuelas de las Ciencias Sociales han elaborado conceptos tanto a lo largo del tiempo como simultáneamente. Dichos conceptos pueden diferir en su carga significativa, poseer pluralidad de significados o ser prácticamente idénticos; un mismo concepto incluso puede presentar significados contradictorios. De esto se deriva que los conceptos, como las propias cosas, poseen una biografía y una vida social. Emergen a partir de invenciones, collages o apropiaciones. Sufren extrapolaciones, transformaciones, difusiones, desgastes, devaluaciones, e incluso pueden ser estigmatizados o constituirse en lugares comunes; desaparecen para volver a resurgir. Esta biografía, en ocasiones, es olvidada, ignorada o negada por quienes los desarrollan y usan (Menéndez 1999). El uso y desuso de conceptos se encuentra teñido por los oscilantes vaivenes de la memoria: recuerdos y olvidos selectivos los entraman. Por otra parte, las demandas académicas y la propia práctica científica pueden hacer del entramado conceptual un verdadero campo de batalla en donde la 
aceptación y uso por parte de la mayoría posiciona y confiere prestigio a sus creadores/impulsadores. Esta condición dinamiza y acumula estratos significativos en los diversos conceptos esgrimidos. Aquel autor sostiene que los conceptos deben crearse en torno a problemas a abordarse, por lo tanto constituyen instrumentos que van articulando teorías y sufriendo transformaciones (resignificaciones teóricas varias) en relación a su uso. Lejos de constituir esencias o cristalizaciones que deben conservarse, son dinámicos, dado que frente a la emergencia de nuevos problemas, la propia historia y práctica científica así lo requiere. Esto implica que a la hora de utilizarlos resulta necesaria una apropiada definición que se vincule a un problema de investigación, siendo necesaria también una revisión histórica y crítica de los mismos (Menéndez 1999: 155).

Aquí simplemente consideraremos que los conceptos cultura material, materialidad y tecnología corresponden a aquello que se entiende -al interior de la comunidad científica- cuando se usa cada una de las correspondientes expresiones: "cultura material", "materialidad" y "tecnología". Dicho de otro modo, los conceptos mencionados no son ni las palabras (términos de la jerga disciplinar) ni las cosas (o cualquier otra entidad a la que hagan referencia específica, que puede existir o no), sino el "intermediario neutral" entre ellas (sensu Weitz 1977: 8). Estos términos, que forman parte de la toponimia de la geografía antropológica, arqueológica e histórica son liminales o llanamente atraviesan las fronteras disciplinarias. Contemplados así en el plano del lenguaje, pueden ser considerados teóricos, a la vez que observacionales; son parte integral de las teorías de cada disciplina social y, además, refieren a la experiencia sensorial bajo estudio (Gianella 1995: 32).

Según Frege (1903), la definición de un concepto -por medio del enunciado de sus condiciones necesarias y suficientes- es tarea primordial para que este sea considerado como tal. Este autor y muchos otros filósofos, siguiendo la tradición platónica, han sostenido que los conceptos deben ser cerrados, a fin de posibilitar la inteligibilidad del discurso. Sin embargo, algunos de los conceptos ampliamente utilizados en ciencias sociales, no pueden ser definidos en sentido estricto. Éstos son denominados abiertos; es decir que no están gobernados, ni necesitan estarlo, por un conjunto definitivo de criterios (Weitz 1977: 19). Esta suerte de carácter no definitivo se aprecia de forma notoria en las diferentes connotaciones de un mismo recurso lingüístico, según el marco teórico particular -y la matriz disciplinaria, tal como la definió Kuhn- y el uso dado a este (e.g. como término teórico, observacional e incluso como referencia a un tipo de programa de investigación).

Los conceptos aquí discutidos son parte componente de -o suponen-particulares teorías (antropológicas, sociológicas o históricas) sobre el comportamiento humano. Su utilización es fundamental para teorizar acerca de este último. En este sentido, los códigos verbales empleados dan cuenta en rasgos generales de cierto posicionamiento del investigador (a modo de presupuestos teóricos) con respecto al tema de estudio desarrollado (Becker 2011: 59). A lo largo del devenir de las disciplinas en cuestión, las teorías - propias y de otros campos de conocimiento- fueron transformándose, e incluso dejándose a un lado. Debido a su carácter abierto, también lo hicieron los conceptos fundamentales asociados a aquellas, oscilando entre posturas fuertes o esencialistas a posturas débiles o elásticas (Brubaker y Cooper 2001). A su vez, en el caso particular aquí expuesto, se notará que ha existido cierto conservadurismo en lo que a los términos respecta, mas no siempre a su contenido. Por ello, el eje de este estudio es eminentemente conceptual; está dedicado a reflexionar sobre $-\mathrm{y}$, a la vez 
precisar o esclarecer- aquello a lo que refiere, según el enfoque de los investigadores, un número conciso de términos.

Los conceptos aquí abordados fueron variando de significación a lo largo del tiempo, pero lejos de constituir instancias superadoras, suelen coexistir en relativa concordia dentro del ámbito académico. Poseen una sedimentación histórica con estratos que se acumulan y otros que se erosionan, dando lugar a una matriz en apariencia armónica. Los términos utilizados adquirieron un carácter polisémico (i.e. un mismo rótulo para designar más de un concepto). La equivocidad (ambigüedad) asociada, en razón de las diversas cargas conceptuales implícitas en cada uno, pueden considerarse fuentes de confusión teórica bajo ciertas circunstancias. Lo anterior se aplica en particular a aquellos casos en que se ha hecho un uso irreflexivo -en algunos casos, como sinónimos- e impreciso - tan sólo por frecuencia de uso- de la terminología. Los problemas que ello supone para la comunicación entre los investigadores, en especial frente a la existencia de diversas posiciones teóricas, son evidentes. De allí la importancia de utilizar, en la medida de lo posible (cuando estén disponibles), términos precisos. Dadas las circunstancias presentes, donde se cuenta con un repertorio exiguo de vocablos, será necesario definir explícitamente aquello que se entiende designado por cada uno de éstos. En algunos casos, como se verá más adelante, en principio bastará con explicitar la teoría bajo la cual se enmarca.

En función de lo expuesto anteriormente, y tal como hemos anticipado, en los siguientes acápites caracterizaremos los conceptos de interés: tecnología, cultura material y materialidad.

\section{Tecnología}

Los estudios sobre la tecnología han tenido una larga trayectoria desde las postrimerías del siglo XIX y principios del siguiente. No es de extrañar el lugar que ocupó en las obras de reconocidos científicos sociales, dada la importancia que ha tenido históricamente para el ser humano (Geselowitz 1993: 231).

En el campo de la Historia, las obras literarias sobre la historia de las invenciones aparecieron tan tempranamente como el siglo XV. No obstante, hasta principios del siglo XX la mayoría de estos estudios consistieron en cronologías técnicas específicas, manuales y enciclopedias escritos por ingenieros e inventores, que raramente consideraron los aspectos sociales alrededor de la emergencia de la tecnología. Pensadores como Abbott Payson Usher, Lewis Mumford y Sigfried Giedion tomaron la dimensión moral y humana de la tecnología en su sentido más amplio y la consideraron como un fenómeno esencialmente humano más que un proceso independiente. La realidad de su época los llevó a cuestionarse en qué modo influía la tecnología en la sociedad y la cultura del momento, pero consideraron que con un control y dirección adecuados, aquella sería el eje de la reconciliación entre las necesidades del espíritu humano y las condiciones materiales de existencia (Molella 1988).

En el ámbito de la Sociología, la preocupación por la tecnología tampoco quedó al margen de las agendas de investigación desde sus propios inicios. Una forma de entender la tecnología es en función de la actividad productiva. Según Marx, las tecnologías, que él denominó como productos, son el resultado de la adaptación de los frutos de la naturaleza a las necesidades humanas por medio de la producción. Estos productos se constituyen como tales en el acto del consumo (del uso). Este crea la 
necesidad de una nueva producción, al mismo tiempo que esta es necesaria para que exista consumo: ella genera el material para el consumo, la forma de consumo y el sujeto consumidor (Marx 1987). Con posterioridad, algunos investigadores adoptarán una postura centrada en la utilidad para la que fueron hechos los objetos, impresa desde su producción, mientras que otros harán hincapié en el significado y rol de los artefactos en función de su puesta en acto dentro de las relaciones interpersonales, durante su período de consumo (ver más abajo).

El campo conocido como Sociología de la tecnología (o estudio social de la tecnología) es relativamente reciente. Los trabajos más importantes fueron elaborados a partir de la década de 1980. En líneas generales, estos estudios ahondan en los complejos procesos de interacción y configuración recíproca entre la sociedad y la tecnología. Las principales perspectivas son conocidas como: el enfoque de sistemas, el constructivismo social y la teoría del actor-red (Aibar 1996). Según este autor, estos estudios realizan una crítica profunda a dos aspectos fundamentales del determinismo tecnológico. Por un lado, la idea de que la tecnología constituye un ámbito con una lógica propia, cuyo desarrollo es inevitable e independiente de la sociedad. Por el otro, que la tecnología influye de forma significativa sobre esta última, como un factor externo (determinismo tecnológico, sensu stricto). A esta posición se encuentra asociada la idea del desarrollo lineal de la tecnología (sucesión de etapas conectadas en un sentido único). Los análisis constructivistas, en su lugar, harán hincapié en los factores sociales de diverso orden (e.g. económico, político, cultural) que modelan a la tecnología. Consideran además los efectos que esta tiene sobre la sociedad, pero no los entienden como resultado de características inherentes, sino como parte de la interrelación de ambas. No se concede un lugar privilegiado a lo social (determinismo social). Antes, el énfasis está puesto en las interacciones entre la sociedad y la tecnología como parte de una dinámica de configuración mutua (e.g. Pfaffenberger 1992; Klein y Kleinman 2002).

Con relación a la Antropología, los estudios científicos sobre la naturaleza del ser humano, en cuanto sus aspectos físicos, intelectuales y morales, comenzaron a desarrollarse en el siglo XVIII (Bilbao 1991). Durante esta época y, sobre todo, durante la primera mitad del siglo XIX, las ideas de progreso asociadas a la historia humana fueron ganando espacio. Se hizo hincapié ante todo en las cualidades excepcionales y superioridad de nuestra especie respecto del resto de los animales. En los escritos de varios profesionales franceses (e.g. Louis-François Jauffret y Joseph-Marie Degérando) que desde temprano abogaron por un estudio situado de los pueblos salvajes, vislumbramos algunas consideraciones que luego serían desarrolladas por otros.

La noción de progreso estuvo estrechamente asociada a la perspectiva evolucionista, que hundía sus raíces en el siglo anterior. Los cambios en una sociedad fueron vistos como parte de una secuencia de estadios evolutivos, de lo simple a lo complejo (e.g. salvajismo, barbarie y civilización, por citar la secuencia más conocida). Estas instancias se caracterizaban por una suma de rasgos, que en su conjunto las definían y servían como indicadores del grado de avance para comparar sociedades pasadas y contemporáneas. Estaban conectadas entre sí, sin solución de continuidad, de modo unilineal y unidireccional. El ser humano, a lo largo de su historia había transitado una tras otra hasta alcanzar la máxima expresión de racionalidad: la sociedad occidental decimonónica (algunos acotaron esta posición a la sociedad británica). Este camino era inevitable, formaba parte de una dinámica universal del desarrollo de la naturaleza y del ser humano. 
Los antropólogos y otros científicos sociales de la segunda mitad del siglo XIX (e.g. Morgan, Taylor, Spencer, Bachofen) abrevaron en estas ideas. En esta época, a la luz de los profundos cambios asociados a la industrialización en Europa, especialmente en Inglaterra, la tecnología fue vista como la expresión patente de los logros alcanzados únicamente por la sociedad occidental. Así, pasó a ocupar un lugar preponderante -en la academia y para el vulgo, que aún gusta emplear este criterio- para definir el progreso. En la primera parte de La Sociedad Primitiva, titulada 〈Desenvolvimiento de la inteligencia a través de invenciones y descubrimientos», Morgan sostuvo: "recomponiendo las diversas trayectorias del progreso hacia las edades primitivas del hombre, esperando una de otras según el orden de aparición de los inventos y hallazgos por un lado, e instituciones por otro, comprendemos que aquellos mantienen entre sí un vínculo progresivo y estos una relación de desenvolvimiento" (Morgan 1970: 9).

En esta misma sección Morgan definió las características de los diferentes grados de progreso del hombre. Adoptó los períodos salvajismo, barbarie (que subdividió en inferior, medio y superior) y civilización, que distinguió principalmente sobre la base de la presencia/ausencia de implementos y artes de subsistencia. Cada uno de estos estadios representaba una cultura diferente y un modo de vida más o menos particular (Morgan 1970).

Las secuencias estrictamente tecnológicas (e.g. las edades de Piedra, Bronce y Hierro, definidas por el historiador danés Christian Jürgensen Thomsen) no fueron privativas del evolucionismo, aunque con él adquirieron un sentido especial y alcanzaron categoría teórica. Las duras críticas recibidas por la escuela norteamericana conocida como particularismo histórico y el funcionalismo británico durante las primeras décadas del siglo XX, dieron como resultado el abandono de muchos de los supuestos y proposiciones previas (ver Harris 2009; Lischetti 2014; entre otros, para mayor información sobre la historia del pensamiento antropológico).

Precisamente, la Antropología de la primera mitad del siglo XX también provee un marco para dar cuenta de la articulación entre la tecnología y la sociedad. No obstante, cabe resaltar que durante esta etapa la disciplina estuvo focalizada en los proyectos etnográficos. Y dentro de este escenario, la tecnología ocupó un lugar subordinado. Como excepción de lo anterior podemos situar a la temprana propuesta sociológica-antropológica de Marcel Mauss, que tuvo profundas implicaciones en los demás antropólogos (en especial franceses) tiempo más tarde. En su trabajo Les techniques du corps (1934), Mauss destacó que las técnicas son, por encima de cualquier otra cosa, una producción social. Algo distintivo de su enfoque fue que, según argumentó, muchos de los actos más cotidianos o naturales de las personas -en las que no necesariamente se ven involucrados objetos- están altamente socializados. Los objetos son partes constituyentes del "hecho social total", productos y técnicas impuestas a los individuos por una tradición o estructura social existente con anterioridad al individuo. Esta obra constituyó la base de los posteriores estudios sobre tecnología, aunque este campo debió esperar alrededor de medio siglo para ser desarrollado (Lemonnier 1992: 1).

La tecnología -o las técnicas, término que se utiliza indistintamente dentro de esta corriente antropológica- comprende: "todos los aspectos del proceso de acción sobre la materia, sea esto resacarse la nariz, plantar batatas, o fabricar jumbo jets" (Lemonnier 1992: 1; traducción de Andrés Laguens). En la línea conceptual de la escuela francesa, que sienta sus bases en las ideas propuestas por Mauss, Lemonnier 
sostuvo que las tecnologías no son sólo cosas y los medios utilizados por la sociedad para actuar sobre la materia, sino que $-\mathrm{y}$ diremos en este punto, al igual que para muchos otros etnólogos, arqueólogos e historiadores- son producciones sociales en sí mismas. Los artefactos deben ser considerados por lo que son: sólo una parte de la tecnología. A pesar de ello: "la gran masa de estudios (...) han estado dedicados a los artefactos, y sólo a los artefactos" (Lemonnier 1992: 4).

Esta afirmación deja entrever el fuerte sesgo material que existe en detrimento del estudio de los demás aspectos socioculturales íntimamente relacionados. Continuando con la propuesta de este autor, las técnicas presentan tres niveles de interacción que le otorgan al conjunto un carácter sistémico (technical system): 1) una técnica está formada siempre por múltiples componentes: las secuencias de acción, las herramientas (incluyendo al cuerpo humano), los gestos, las fuentes de energía, los actores y las representaciones; ellos forman un sistema, en el sentido que si uno cambia puede suceder un cambio en uno o varios de los otros (i.e. cada técnica es el locus de múltiples interacciones y de constantes ajustes entre sus elementos); 2) en una sociedad dada en un momento determinado, varias técnicas están relacionadas unas con otras de varias formas y por varios motivos; 3 ) un sistema técnico es siempre parte de un todo socio-cultural que lo incluye, de modo que se encuentran sujetos a transformaciones recíprocas. Es importante tener en cuenta que la forma en que un objeto es fabricado, usado o intercambiado está relacionada con sistemas de prácticas y creencias que van más allá de los aspectos estrictamente materiales (Lemonnier 1986, 1993, 2003).

Es indudable que la Arqueología, en tanto campo de investigación que se ocupa de estudiar el comportamiento humano sobre la base de los restos materiales, evidencia por excelencia de sus actividades pasadas (Trigger 1992), posee un lugar privilegiado en lo que al análisis de la tecnología respecta. No obstante, los aportes teóricos en torno a esta cuestión fueron durante mucho tiempo comparativamente escuetos con respecto al de otras disciplinas.

En sus inicios decimonónicos, la orientación de la Arqueología con respecto a los materiales estuvo más bien caracterizada por la lógica -imperante desde tiempo atrás- propia del coleccionismo. La preocupación fundamental radicaba en establecer secuencias ordenadas de los restos hallados; la descripción e identificación de artefactos era (y sigue siendo) la base de cualquier análisis ulterior. Adam Smith había establecido en 1762 un sistema escalonado que los arqueólogos incorporarían un siglo más tarde para organizar y clasificar los instrumentos líticos, las vasijas y los bronces, según una secuencia evolutiva y progresiva, acorde a las teorías imperantes con relación al progreso humano (ver más abajo). Al respecto, Gamble destacó que este sesgo ha perdurado hasta la actualidad, donde: "la medida del progreso sigue siendo la tecnología, hasta el punto de que no importa si sabes conducir o no, sino qué es lo que conduces" (Gamble 1994: 12).

Desde fines del siglo XIX y durante la primera mitad del siguiente, predominaron varias orientaciones que en conjunto se pueden englobar como parte de la Arqueología tradicional -histórico-cultural o normativa, según sus denominaciones a posterio$r i$-que hizo hincapié en el estudio de las particularidades de las diversas culturas. Los restos arqueológicos se consideraron definitorios de los tipos culturales: en el caso norteamericano, estos se clasificaron de acuerdo con la presencia de cierto conjunto distintivos de artefactos (e.g. tipos de instrumentos líticos o cerámicos, equiparables con los rasgos etnográficos), mientras que entre los investigadores europeos primó 
la organización de las distintas culturas de acuerdo con la presencia de frecuencias de artefactos formalmente clasificados, es decir que dos conjuntos se comparaban a partir de las cantidades relativas de los distintos tipos de artefactos (Binford y Sabloff 1982). En la mayoría de los casos, la tecnología se consideró a grandes rasgos como un reflejo directo de las ideas y normas de la sociedad, relativamente homogénea y estable al interior de cada cultura, sujeta a procesos de cambio anclados principalmente en el difusionismo. En Europa, por ejemplo en la escuela francesa, podemos resaltar el aporte que hizo Leroi-Gourhan desde la década de 1960 (e.g. Leroi-Gourhan 1971, 1988, 1989), que abordó estas cuestiones desde enfoques teóricos y de método sobre la base de temas como la tecnología y el arte, principalmente.

En EE.UU. y otras regiones, la propuesta de Binford (1962) tuvo fuertes repercusiones. Esta se orientó hacia la explicación más que la explicitación de acontecimientos específicos -característica de la orientación histórica, que continuó prevaleciendo en Europa- y a la consideración de la cultura como un sistema compuesto por múltiples subsistemas (social, tecnológico e ideológico) interrelacionados. Dentro de este enfoque, la relación con el medio exterior se volvió crucial para explicar el funcionamiento de las sociedades. El énfasis puesto en los diversos aspectos componentes de cada cultura, quedó soslayado por una orientación fuertemente materialista, por lo que la mayoría de los estudios que seguirían por este camino se preocuparían en particular por los artefactos "tecnómicos"-la tecnología, entonces, se entiende de un modo restringido- aquellos que poseen su contexto funcional primario en contacto directo con el ambiente físico.

Es notable destacar que existe un gradiente de dificultad en la interpretación de los datos, de acuerdo con el tipo de aspectos que se consideren. Así, la tecnología es la categoría más fácil, y le siguen en orden creciente de dificultad la economía (fundamentalmente la subsistencia), la organización social y política, y finalmente la ideología (Hawkes 1954, citado en Trigger 1992: 362). Una corriente importante de estudios dentro de la Arqueología se relaciona con la organización de la tecnología de sociedades de cazadores-recolectores. Por caso, Nelson (1991) analizó las diferentes estrategias de resolución de problemas que desarrolla el ser humano en el interjuego con el ambiente (tecnologías conservadas, expeditivas y oportunísticas), mientras que Bamforth (1986) examinó distintos aspectos y contextos de aplicación del comportamiento de conservación, en particular para el caso de los instrumentos líticos.

La consideración inherentemente social de la tecnología fue paulatinamente reconocida dentro de la comunidad académica. Pero el estudio de dichos aspectos socioculturales fue abordado de forma integral años más tarde, sobre todo a partir de los intereses cognitivos de los arqueólogos procesuales (a partir de la década de 1970), así como por el programa propuesto por algunos dentro de la denominada Arqueología post-procesual. Estas diversas aproximaciones fueron muy diferentes entre sí: los primeros siguieron dentro del marco de rigurosidad metodológica de la Arqueología procesual, mientras que los segundos se volcaron por análisis de tipo hermenéutico y fenomenológico, aunque compartieron su interés por concretar la práctica de una Arqueología más holística (Flannery y Marcus 1998).

El rol activo de los individuos dentro de la configuración de la sociedad pronto cobró visibilidad dentro de las propuestas de algunos arqueólogos. Esta idea de transformación hunde sus raíces en la teoría marxista. Dentro de este contexto, los objetos dejaron de ser considerados como un producto directo del comportamiento humano, 
un reflejo pasivo del sistema sociocultural, y pasaron a verse mediatizados por las ideas, creencias y significados de individuos dentro de un contexto histórico-cultural específico (Hodder 1994). Desde diversas perspectivas teóricas, varios autores contribuyeron con la comprensión de los procesos de significación. Al respecto, Renfrew destacó que durante las últimas décadas del siglo pasado se pudo apreciar una creciente articulación entre los estudios sobre tecnología en Arqueología y Antropología Social, que redundó en una contribución mutua. Este referente de la denominada Arqueología cognitiva, se interesó en los últimos años por el involucramiento humano con el mundo material a lo largo de su historia (Renfrew 2001) ${ }^{6}$.

Dentro del ámbito antropológico y arqueológico, tal como hemos anticipado, otro de los conceptos largamente utilizados desde sus inicios como disciplina fue el de cultura material. De este último nos ocuparemos a continuación.

\section{Cultura material}

Al igual que el concepto de tecnología, el de cultura material no constituye una categoría natural, sino que tiene su propia genealogía (Preucel y Meskell 2004). Este ha sido abordado desde distintas disciplinas, tales como la Sociología (e.g. Germani 1955), la Historia (e.g. Braudel 1984; Burke 2001), el Arte y la Estética (e.g. van Lier 1971), entre las principales. Sin embargo, las temáticas concernientes a los objetos de producción humana pueden considerarse relativamente marginales frente al gran corpus de investigaciones desarrolladas en ciencias sociales (e.g. Olsen 2003; Latour 2008; Rodríguez 2006; Mills y Walker 2008). No obstante, a diferencia de lo que sucedió en los citados campos de conocimiento, dentro de la Antropología y, más específicamente, la Arqueología, este tipo de análisis ocupó un espacio significativo. Esta situación se debe a que dicha disciplina convive con el análisis de materiales como parte de su praxis. De esta forma, no es extraño que las cosas (artefactos u objetos) hayan sido teóricamente conceptualizadas. Es por ello que frente a la contemporánea revitalización de los estudios de cultura material en el ámbito de la arqueología anglosajona se haya propuesto a esta disciplina a la vanguardia de dichos estudios (Rodríguez 2006).

Existen numerosas definiciones de cultura material. A pesar de los matices, las distintas perspectivas desarrolladas en el marco de las diversas disciplinas, pueden asumirse semejantes en tanto y en cuanto se consideren los principales lineamientos, que giran en rededor de: 1) cómo las sociedades y las personas clasifican los objetos que producen, consumen, intercambian y descartan; 2) el rol de los objetos en las relaciones, prácticas y representaciones culturales y 3 ) el poder simbólico (como expresión de ideas, valores, etc.) así como el papel que desempeñan en los conflictos y cambios sociales.

Este concepto comienza a utilizarse en Inglaterra a mediados del siglo XIX, principalmente dentro del campo de la Antropología y Arqueología, ligado al desarrollo de museos. Se empleó en un principio para categorizar los objetos vinculados a proyectos culturales relacionados con la sofisticación social de grupos pretéritos,

Otros se enfocaron en el proceso de materialización de la ideología -entendida como una fuente de poder socialy en la posibilidad de estudiar los procesos de competencia, control y negociación de las relaciones al interior de la sociedad por intermedio de las distintas expresiones materiales (DeMarrais et al. 1996). 
i.e. evolucionismo (Buchli 2002). Desde fines del siglo XIX y principios del XX, el estudio de la producción y uso de objetos por parte de las diversas sociedades que habitaban los territorios coloniales fue parte del núcleo de la disciplina antropológica emergente. Aquí se enfatizó la relación isomórfica, estrecha y directa entre objetos y culturas (entendidas como pueblos o grupos humanos). Dentro de esta postura clásica de la Antropología, según Miller (1987) la investigación de la cultura material se redujo a dos áreas periféricas. Por un lado a aquellos estudios que focalizan en lo esotérico y exótico de los artefactos y por ende en la distancia entre los hombres y su ambiente. Por el otro a comprender a la tecnología dentro de estudios generales sobre la evolución, el progreso y la difusión de las sociedades humanas.

Cancino Salas (1999) agrupa las concepciones que sobre la cultura material proponen diversas disciplinas en dos categorías: clásicas y no clásicas. Los diversos enfoques englobados en la concepción clásica de los estudios sobre cultura material poseen en común el hecho de posicionarse epistemológicamente frente a un objeto que puede caracterizarse como neutro. La condición neutral del objeto se manifiesta cuando los mismos son entendidos como cosas separadas e independientes de los sujetos que los construyen, usan, intercambian, comercian y descartan. El evolucionismo y el difusionismo con sus esquemas taxonómicos de las sociedades, sus clasificaciones interétnicas y sus tipologías artefactuales hicieron especial hincapié en los aspectos morfológicos y funcionales más que en aquellos relacionales y simbólicos. Por caso, en el marco de la escuela norteamericana conocida como particularismo histórico, Franz Boas empleó el término cultura material para hacer referencia de forma genérica al conjunto artefactual de las sociedades (Boas 1964).

Por otro lado, el objeto es considerado no neutro cuando se lo entiende cumpliendo un rol activo y pleno en su relación con los sujetos y con otros objetos. Esta perspectiva en torno a los objetos puede continuar con enfoques clasificatorios o desarrollar enfoques de-constructivistas de los mismos. Pueden enumerarse distintas perspectivas dentro de esta aproximación. Por citar sólo algunos ejemplos, en Antropología destacan: 1) los estudios de los sistemas de clasificación de bienes de Douglas e Isherwood (1990); 2) los estudios desarrollados en torno a la vida social de las cosas y a la biografia cultural de las cosas de Appadurai (1986) y Koppitoff (1986), respectivamente $^{7}$; y 3) los estudios culturales del consumo con exponentes de la talla de McCraken (1986), Miller (1987, 1998) y García Canclini (1995), entre otros.

En Arqueología, desde la década del ochenta y dentro de la aproximación no neutral de la cultura material se destacan varias perspectivas. En primer lugar, resaltan a aquellas que enfatizan el carácter ideológico del objeto. Esta perspectiva posee una clara influencia de pensadores como Karl Marx, Antonio Gramsci, Lois Althusser,

\footnotetext{
Durante las últimas décadas se hizo hincapié en la vinculación entre las personas y los objetos a lo largo de la historia de cada uno de ellos. Más allá de los acercamientos sobre la vida de uso (use-life approaches) de los artefactos, focalizados en los cambios morfológicos y funcionales, se ubica la perspectiva de Gosden y Marshall (1999). Según esta perspectiva, las personas y los objetos sufren constantes transformaciones que se encuentran mutuamente vinculadas. Los referidos autores proponen el estudio de la biografía cultural de los objetos desde diversos acercamientos (e.g. a partir de la observación de las alteraciones físicas de las piezas, de los contextos de intercambio o del análisis de determinada performance) para elucidar el proceso de interacción social a partir del cual se crean, negocian, e incluso reconfiguran, los significados y valores de las cosas (Gosden y Marshall 1999). En otro trabajo, Gosden (2005) se centró en la agencia de los objetos, en el rol activo de determinados grupos de artefactos -que crean universos estilísticos con una lógica exclusiva- sobre las personas que los producen y hacen uso de ellos.
} 
Herbert Marcuse y Jürgen Habermas (Leone 1988, 1995). En ella, la cultura material se convierte en expresión de poder y de dominación social de las elites. En segundo lugar, la perspectiva semiótica de la cultura material (vinculada al denominado giro lingüístico en las ciencias sociales) cuyas influencias se remontan a las obras de Ferdinand de Saussure, Charles Sanders Pierce, Roland Barthes, Victor Turner, Erving Goffman y Jacques Derrida, entre otros. Dicha perspectiva -la más prolífica en producción- considera a la cultura material como cargada de significado, un texto a ser leído, un medio comunicativo no verbal, resaltando sus aspectos simbólicos (e.g. Hodder 1982, 1994; Shanks y Tilley 1992; Beaudry et al. 1996). Dicho significado es negociado en cada contexto por los distintos agentes, sectores, estamentos o clases sociales tanto del pasado como del presente. Al ser parte constitutiva de esta relación de negociación, la cultura material desempeña un rol importante y activo en el control, condicionamiento y mantenimiento social e incluso en su cambio. En tercer lugar, desde finales de la década del noventa comenzó a generarse un profuso aumento de trabajos arqueológicos influidos por diversos enfoques de la teoría social contemporánea; por ejemplo, la teoría de las prácticas, de la estructuración, la fenomenología, la hermenéutica y la teoría del actor-red. Pierre Bourdieu, Anthony Giddens, Michel Foucault, Michel de Certeau, Charles Ortner, Marshall Sahlinns, Paul Ricouer, Bruno Latour y Alfred Gell, entre otros son los pensadores cuyas ideas, teorías, conceptos y modelos han servido de base para entender las complejas interacciones entre los grupos sociales y su cultura material en diferentes escalas temporales y espaciales. A partir de ellas fueron abordadas diferentes temáticas: género, clase, cambio cultural, desigualdad y diferenciación social. Estos diversos y eclécticos contenidos atribuidos a la cultura material pueden englobarse en lo que Rodríguez Corral (2006) denomina materialidad arqueológica post-procesual, en clara alusión a dicha corriente.

En las postrimerías del siglo XX, en estrecha relación con esta última concepción de la cultura material comenzó a emplearse el término materialidad, sobre el que versaremos en el acápite que sigue.

\section{Materialidad}

Tímidamente desde la segunda mitad de la década del noventa del siglo XX, pero con gran énfasis hacia la segunda mitad de la primera década del siglo XXI, comenzó a establecerse un nuevo debate en torno al concepto de cultura material. Diversos arqueólogos y antropólogos del mundo anglosajón comenzaron a utilizar el uso del concepto de materialidad en detrimento del de cultura material (e.g. Preucel y Meskell 2004; Jones 2004; Meskell 2005; Miller 2005; Mills y Walker 2008; Pollard 2008). Si bien el término materialidad ha sido desde entonces utilizado más asiduamente en las producciones arqueológicas, aún existe poca especificidad en torno a su definición (Mills y Walker 2008). Esta situación se manifiesta en la convivencia de los conceptos de cultura material y materialidad, que se percibe en diversos trabajos que no manifiestan una ruptura o clara diferencia entre ambos (e.g. Hodder 2004; Preucel y Meskell 2004; Rodríguez 2006; Smith 2007). Por otra parte, otros autores intentaron evidenciar las diferencias entre ambos conceptos (e.g. Olsen 2003; Miller 2005; Mills y Walker 2008). Pueden apreciarse en dichos investigadores dos críticas no excluyentes; el concepto de materialidad: 1) enfatiza en las relaciones intrínsecas 
entre la gente y sus prácticas, los objetos, el espacio y el tiempo; y 2) hace hincapié en la eliminación del término cultura.

Con respecto a la primera postura, según Meskell (1998, 2004, 2005) los estudios arqueológicos basados en la materialidad divergen de forma significativa con los estudios convencionales de la cultura material. Esta autora considera que la perspectiva empírica de los estudios de cultura material se centra en los análisis morfológicos, constructivos o en los tipos de materiales empleados, constituyendo una "tendencia empírica", sin estar obligados a verlos inmersos en las relaciones sociales. Por otra parte, la "tendencia teórica" de los estudios de la cultura material toma al artefacto como epifenómeno, enfatizando en su rol de expresiones culturales (simbólicas, ideológicas) desde diversos enfoques interpretativos. Meskell considera que los estudios de la materialidad exceden los objetos per se, los mismos sólo pueden comprenderse en una interrelación triádica entre sociabilidad, temporalidad y espacialidad (Meskell 2004, 2005), constituyendo una instancia superadora de las previas posturas dicotómicas (Vaquer 2012). Consideramos que ambos conceptos exhiben mayor cantidad de características comunes que diferencias. Por ejemplo, dentro de los denominados nuevos estudios de la cultura material, Miller y Tilley consideraron que: "los estudios de cultura material podrían ser definidos en términos generales como la relación entre las personas y las cosas con independencia del tiempo y el espacio" (Miller y Tilley 1996, citado en Cochran y Beaudry 2006: 197; la traducción es personal).

Esta definición, podemos decir, no evidencia grandes diferencias con las postuladas por Meskell. Por otra parte, estos investigadores proponen que los estudios de la materialidad deben ser contextualizados temporal, espacial y socialmente ateniéndose a cada caso en particular y que los arqueólogos deben prestar atención a los momentos de producción, intercambio, uso, consumo y descarte. La contextualización en las investigaciones arqueológicas no constituye prerrogativas de los estudios de la materialidad. Los estudios de la cultural material hicieron hincapié en ello, desde al menos principios de la década de 1990 (e.g. Beaudry et al. 1996; Orser 1996, 2000). Consideramos que las críticas realizadas por los investigadores que abogan por el concepto de materialidad parecen haber sido realizadas contra la postura clásica de la cultura material -aquella que Ingold (2013) considera como hibernante en los sótanos de la museología-, en donde el objeto es considerado neutro y puede ser analizado separado de su contexto socio-cultural de producción, uso, consumo y descarte (Cancino 1999), más que contra la mirada de los nuevos estudios de la cultura material.

Con respecto a la segunda postura, sólo unos pocos autores consideran importante eliminar la palabra cultura, para dar cuenta de los estudios sobre materialidad (e.g. Ingold 2000; Olsen 2003). La ambigüedad del concepto cultura (sobre el cual se han vertido océanos de tinta) puede generar confusión cuando se lo entiende desde la ligadura entre lo intangible y lo material. Si bien las críticas por parte de los estudios de la materialidad con respecto a este binomio conceptual no son profusas, pueden estar relacionadas a su postura que propone que todo estudio de la materialidad debe ser contextualizado temporal, espacial y socialmente. Esta perspectiva particularista entraría en choque con la concepción universalista en torno a la cultura material. Otra critica que podría llegar a hacérsele al concepto de cultura ligado al mundo material está relacionada con una concepción esencialista y normativa de la cultura, en donde lo material se vería como propio y definitivo de grupos culturales cerrados y estáticos 
Cabe destacar lo que consideramos novedoso del enfoque sobre la materialidad. Si bien desde la última década del siglo XX los denominados nuevos estudios de la cultura material comenzaron a emplear conceptos desarrollados por la teoría social y dentro de estos especialmente aquellos ligados a las teorías de la praxis, los estudios de la materialidad específicamente dan cuenta de su uso en sus definiciones. Por ejemplo, en palabras de Nielsen: "la noción de materialidad, entendida como la dimensión material de la práctica (acción social situada cultural e históricamente) resalta los procesos a través de los cuales los materiales (artefactos, monumentos, paisajes, etc.) y los agentes humanos, objetos y sujetos se constituyen recíprocamente" (Nielsen 2008: 208; la traducción es personal).

Basados en la ruptura del binomio dicotómico sujeto/objeto (propio de las filosofías idealistas de Descartes) los estudios de la materialidad buscan poder apreciar: "cómo la gente y los objetos se constituyen mutuamente y funcionan juntos como formas híbridas" (Pollard 2008: 46).

En definitiva, la materialidad enfatiza las formas o maneras en que los objetos se consustancian en las prácticas sociales. Los aspectos físicos del artefacto se encuentran entrelazados profundamente en la praxis que da forma a la experiencia humana. La materialidad es indispensable para rastrear, pensar y comprender las relaciones sociales de los grupos humanos del pasado y del presente. Su investigación sociológica, histórica, arqueológica o antropológica puede y debe llevarse a cabo a pesar de la fragmentariedad de los registros empleados. El estudio de la materialidad implica entonces analizar los vínculos establecidos entre los hombres, las cosas y su entorno así como las relaciones sociales que entre ellos median y los constituyen.

\section{Consideraciones finales}

En este trabajo realizamos un estado de la cuestión sobre el devenir conceptual de tres conceptos ¿nociones? que fueron acuñados y desarrollados en múltiples Ciencias Sociales, particularmente en Antropología, Arqueología y disciplinas afines desde finales de la centuria decimonónica: tecnología, cultura material y materialidad. Dichos conceptos, desde diversos enfoques teóricos, tratan de abordar la transformación producida por los humanos sobre la materia aportada por la naturaleza. Los pensadores que desarrollaron y usaron estos conceptos han tratado de arrojar luz sobre diversos interrogantes: ¿cómo nos relacionamos a través de las cosas?, ¿los objetos son solo materiales?, ¿qué significan y por qué?, ¿poseen agencia?, entre otros. A través de ellos, se buscó conocer las características del mundo material de las sociedades pretéritas y actuales.

Dentro de cada una de las disciplinas mencionadas y a lo largo de su desarrollo como campos del conocimiento, los conceptos de tecnología, cultura material y materialidad han resultado de utilidad para pensar el pasado y presente de las sociedades bajo estudio. A lo largo de los diversos devenires disciplinares, los tres conceptos caracterizados han sido objeto de cambios tanto en su significación como en su uso práctico. En razón de esto último, con el fin de poder apreciar y comprender dichas mutaciones, fue necesario debatir sobre los procesos de conceptualización tanto al interior de las disciplinas como a nivel interdisciplinar. El análisis descriptivo, interpretativo y contextual que aquí esbozamos, conforme a perspectivas teóricas particulares y vínculos allende las fronteras disciplinares, 
reviste especial interés para comprender el alcance y las limitaciones a la hora de utilidad cada uno de los conceptos.

Independientemente del lugar que se le ha otorgado a cada uno de los conceptos, creemos haber expuesto con nitidez que en estos se entraman de forma variable los materiales, los individuos o sociedades, los espacios habitados y los conocimientos generados y empleados. Sus adscripciones teóricas, sus variaciones, así como su uso o abandono, solo serán entendidas dentro de los contextos históricos de su producción. Comprender su historicidad permite asumir con plena consciencia cierto posicionamiento teórico, en este caso, con respecto a los modos de comprender la ingente diversidad de producciones materiales y su papel dentro de las sociedades estudiadas. A lo largo del escrito hemos visto que, a nivel conceptual, existen solapamientos. De allí que las propuestas teóricas de los distintos campos de las Ciencias Sociales pueden verse enriquecidas mutuamente. Entre estos puntos en común, consideramos interesante señalar la siguiente perspectiva: estos conceptos no refieren ni una entidad empírica con una lógica y fines propios, ni a una mera colección de las producciones concretas de las personas; antes bien, con sus matices, permiten conceptualizar un complejo entramado de conocimientos y relaciones interpersonales dentro del que cobran significación las expresiones materiales producidas y utilizadas al interior de una sociedad en determinadas coordenadas espacio-temporales. Pensar en las cosas no deja nunca de ser un pensar en nosotros.

\section{Referencias}

Aibar, Eduardo. 1996. «La vida social de las máquinas: orígenes, desarrollo y perspectivas actuales en la Sociología de la Tecnología». Reis: Revista Española de Investigaciones Sociológicas 76: 141-170. http://dx.doi.org/10.2307/40183990.

Appadurai, Ardjun. 1986. «Introducción: las mercancías y la política del valor», en La vida social de las cosas. Perspectiva cultural de las mercancías, Ardjun Appadurai, ed., pp. 17-87. México: Grijalbo.

Bamforth, Douglas B. 1986. «Technological Efficiency and Tool Curation». American Antiquity 51 (1): 38-50. http://dx.doi.org/10.2307/280392.

Beaudry, Mary, Lauren Cook y Stephen Mrozowski. 1996. «Artifacts and Active Voices: Material Culture as Social Discourse», en Images of the Recent Past. Readings in Historical Archaeology, Charles E. Orser Jr., ed., pp. 272-310. Walnut Creek: Altamira Press.

Becker, Howard. 2011 (1986). Manual de escritura para cientificos sociales. Buenos Aires: Siglo XXI Editores.

Bilbao, Cristina, comp. 1991. La ciencia del hombre en el siglo XVIII. Jauffret, Cuvier, Degérando y otros. Buenos Aires: Centro Editor de América Latina.

Binford, Lewis R. 1962. "Archaeology as Anthropology». American Antiquity 28 (2): 217225. http://dx.doi.org/10.2307/278380.

Binford, Lewis y Jeremy A. Sabloff. 1982. «Paradigms, Systematics, and Archaeology». Journal of Anthropological Research 38 (2): 137-153. http://dx.doi.org/10.1086/ jar.38.2.3629594

Boas, Franz. 1964 (1911). Cuestiones fundamentales de Antropología Cultural. Buenos Aires: Solar/Hachette.

Braudel, Fernand. 1984 (1979). Civilización material, economía y capitalismo, siglo XV-XVIII, Tomo 1 («Las estructuras de lo cotidiano: lo posible»). Madrid: Alianza Editorial. 
Brubaker, Roger y Frederick Cooper. 2001. «Más allá de la identidad». Apuntes de investigación 7: 30-67.

Buchli, Victor. 2002. The Material Culture Reader. Oxford: Oxford International Publisher.

Burke, Peter. 2001. Visto y no visto. El uso de la imagen como documento histórico. Barcelona: Editorial Crítica.

Cancino Salas, Ronald. 1991. «Perspectivas sobre la cultura material». Anales de Desclasificación 1 (2): 1-20.

Cochran, Matthew D. y Mary C. Beaudry. 2006. «Material Culture Studies and Historical Archaeology», en The Cambridge Companion to Historical Archaeology, Dan Hicks y Mary C. Beaudry, eds., pp. 191-204. Cambridge: Cambridge University Press.

Collins, Randall. 1989. "Toward a Theory of Intellectual Change: The Social Causes of Philosophies». Science, Technology, \& Human Values 14 (2): 107-140.

Coseriu, Eugenio. 1977. Principios de semántica estructural. Madrid: Editorial Gredos.

DeMarrais, Elizabeth, Luis Jaime Castillo y Timothy Earle. 1996. «Ideology, Materialization and Power Strategies». Current Anthropology 37 (1): 15-31. http://dx.doi.org/10.1086/204472.

Díez, José A. y Ulises C. Moulines. 1999. Fundamentos de Filosofía de la Ciencia. Barcelona: Editorial Ariel.

Douglas, Mary y Baron Isherwood. 1990 (1979). El mundo de los bienes. Hacia una antropología del consumo. México: Editorial Grijalbo, CONACULTA.

Flannery, Kent V. y Joyce Marcus. 1998. «Cognitive Archaeology», en Reader in Archaeological Theory. Post-Processual and Cognitive Approaches, David S. Whitley, ed., pp. 35-49. Londres: Routledge.

Frege, Friedrich L.G. 1903. Grundgesetze der Arithmetik, Vol. 2. Jena: Verlag Hermann Pohle.

Gamble, Clive. 1994. «El poblamiento de Europa hace 700.000-40.000 años BP», en Prehistoria de Europa, Barry W. Cunliffe, ed., pp. 11-46. Barcelona: Editorial Crítica.

García Canclini, Néstor. 1995. Consumidores y ciudadanos. Conflictos multiculturales de la globalización. México: Editorial Grijalbo.

Germani, Gino. 1955. La estructura social de la Argentina. Análisis estadístico. Buenos Aires: Raigal.

Geselowitz, Michael N. 1993. «Archaeology and the Social Study of Technological Innovation». Science, Technology, \& Human Values 18 (2): 231-246. http://dx.doi.org/10.1177/016224399301800207

Gianella, Alicia E. 1995. Introducción a la epistemología y la metodología de la ciencia. Buenos Aires: Editorial de la Universidad Nacional de La Plata.

Gosden, Chris. 2005. «What Do Objects Want?» Journal of Archaeological Method and Theory 12 (3): 193-211. http://dx.doi.org/10.1007/s10816-005-6928-x.

Gosden, Chrise e Yvone Marshall. 1999. «The Cultural Biography of Objects». World Archaeology 31 (2): 169-178. http://dx.doi.org/10.1080/00438243.1999.9980439.

Harris, Marvin. 2009 (1968). El desarrollo de la teoría antropológica. Una historia de las teorías de la cultura. Madrid: Siglo XXI Editores.

Hodder, Ian. 1982. Symbols in Action. Cambridge: Cambridge University Press.

- 1994 (1988). Interpretación en Arqueología. Corrientes actuales. Barcelona: Editorial Crítica.

-. 2004. «The 'Social' in Archaeological Theory: An Historical and Contemporary Perspective», en A Companion to Social Archaeology, Lynn Meskell y Robert W. Preucel, eds., pp. 23-42. Malden: Blackwell. 
Ingold, Tim. 2000. The Perception of the Environment. Essays on Livelihood, Dwelling and Skill. Londres y Nueva York: Routledge.

—. 2013. «Los materiales contra la materialidad». Papeles de Trabajo 11: 19-39.

Jones, Andrew M. 2004. "Archaeometry and Materiality: Materials-Based Analysis in Theory and Practice». Archaeometry 46 (3): 327-338. http://dx.doi.org/10.1111/j.14754754.2004.00161.x.

Klein, Hans K. y Daniel L. Kleinman. 2002. «The Social Construction of Technology: Structural Considerations». Science, Technology, \& Human Values 27 (1): 28-52.

Klimovsky, Gregorio. 2005. Las desventuras del conocimiento cientifico. Buenos Aires: A-Z Editora.

Kopytoff, Igor. 1986. «La biografía cultural de las cosas», en La vida social de las cosas. Perspectiva cultural de las mercancias, Ardjun Appadurai, ed., pp. 89-122. México: Editorial Grijalbo.

Kuhn, Thomas S. 1977 (1959). «La tensión esencial: tradición e innovación en la investigación científica», en La tensión esencial. Estudios selectos sobre la tradición y el cambio en el ámbito de la ciencia, Thomas S. Kuhn, ed., pp. 248-262. México: Fondo de Cultura Económica.

Latour, Bruno. 2008 (2005). Reensamblar lo social. Una introducción a la teoría del actor-red. Buenos Aires: Manantial.

Lemonnier, Pierre. 1986. «The Study of Material Culture Today: Toward an Anthropology of Technical Systems». Journal of Anthropological Archaeology 5 (2): 147-186. http:// dx.doi.org/10.1016/0278-4165(86)90012-7.

-. 1992. «Technology and Anthropology», en Elements for an Anthropology of Technology, pp. 1-24. Ann Arbor: Anthropological Papers 88, Museum of Anthropology, University of Michigan.

-. 2003. «Objects, Technical Systems and Systems of Thought». Centre de Recherche et de Documentation sur l'Océanie y Centre National de la Recherche Scientifique. www. necep.net/papers/OS_Lemonnier.pdf.

- - ed. 1993. Technological Choices. Transformation in Material Cultures Since the Neolithic. Londres y Nueva York: Routledge.

Leone, Mark P. 1988. «The Georgian Order as the Order of Merchant Capitalism in Annapolis, Maryland», en The Recovery of Meaning. Historical Archaeology in the Eastern United States, Mark P. Leone y Parker B. Potter, eds., pp. 235-261. Washington, D.C.: Smithsonian Institution Press.

-. 1995. «A Historical Archaeology of Capitalism». American Anthropologist, New Series 97 (2): 251-268. http://dx.doi.org/10.1525/aa.1995.97.2.02a00050.

Leroi-Gourhan, André. 1971 (1964-1965). El gesto y la palabra. Caracas: Publicaciones de la Universidad Central de Venezuela.

-. 1988 (1943). Evolución y técnica, Tomo 1 (El hombre y la materia). Madrid: Taurus Ediciones.

- 1989 (1945). Evolución y técnica, Tomo 2 (El medio y la técnica). Madrid: Taurus Ediciones.

Lier, Henri van. 1971. «Objeto y estética», en Los objetos, Eberhard Wahl et al., eds., pp. 129-152. Buenos Aires: Tiempo Contemporáneo.

Lischetti, Mirtha, comp. 2014. Antropología. Buenos Aires: Eudeba.

Marx, Karl. 1987 (1857). Introducción general a la crítica de la economía política. México: Cuadernos de Pasado y Presente.

Mauss, Marcel. 1934. «Les techniques du corps ». Comunicación presentada en la Société de Psychologie el 17 de mayo de 1934. Publicado como artículo en Journal de Psychologie 32 (3-4): 365-386 (1936). 
McCraken, Grant. 1986. «Culture and Consumption: A Theoretical Account of the Structure and Movement of the Cultural Meaning of Consumer Goods».

Menéndez, Eduardo. 1999. «Uso y desuso de conceptos: ¿dónde quedaron los olvidos?» Alteridades 17: 147-164.

Meskell, Lynn. 1998. «An Archaeology of Social Relations in an Egyptian Village». Journal of Archaeological Method and Theory 5 (3): 209-243. https://www.jstor.org/stable/20177386?seq $=1$

-. 2004. Objects Worlds in Ancient Egypt. Material Biographies Past and Present. Londres y Nueva York: Berg.

—. 2005. «Introduction: Object Orientations», en Archaeologies of Materiality, Lynn Meskell, ed., pp. 1-17. Oxford: Blackwell.

Miller, Daniel. 1987. Material Culture and Mass Consumption. Oxford: Blackwell.

—. 2005. «Materiality: An Introduction», en Materiality (Politics, History and Culture), Daniel Miller, ed., pp. 1-50. Londres: Duke University Press.

—. ed. 1998. Material Cultures: Why some Things Matters. Cambridge: Cambridge University Press.

Mills, Barbara J. y William H. Walker. 2008. «Introduction: Memory, Materiality, and Depositional Practice», en Memory Work. Archaeologies of Material Practices, Barbara J. Mills y William H. Walker, eds., pp. 3-23. Santa Fe: School of American Research Press.

Molella, Arthur P. 1988. «Inventing the History of Invention. Three Big Thinkers Who Placed Technology at the Hearth of History». Bulletin of Science, Technology \& Society 8 (3): 278-286.

Morgan, Lewis H. 1970 (1877). La sociedad primitiva. Madrid: Editorial Ayuso.

Nelson, Margaret. 1991. "The Study of Technological Organization», en Archaeological Method and Theory, Michael B. Schiffer, ed., pp. 57-100. Tucson: University of Arizona Press.

Nielsen, Axel. 2008. «The Materiality of Ancestors. Chullpas and Social Memory in the Late Prehispanic History of the South Andes», en Memory Work. Archaeologies of Material Practices, Barbara J. Mills y William H. Walker, eds., pp. 207-231. Santa Fe: School of American Research Press.

Olsen, Bjørnar. 2003. «Material Culture after Text: Re-Membering Things». Norwegian Archaeological Review 36 (2): 87-104. http://dx.doi.org/10.1080/00293650310000650.

Orser, Charles E. Jr. 1996. A Historical Archaeology of the Modern World. Nueva York y Londres: Plenum Press.

- 2000 (1995). Introducción a la Arqueología Histórica. Buenos Aires: Editorial del Tridente.

Pfaffenberger, Bryan. 1992. «Technological Dramas». Science, Technology \& Human Values 17 (3): 282-312. http://dx.doi.org/10.1177/016224399201700302.

Pollard, Joshua. 2008. «Deposition and Material Agency in the Early Neolithic of Southern Britain», en Memory Work. Archaeologies of Material Practices, Barbara J. Mills y William H. Walker, eds., pp. 41-59. Santa Fe: School of American Research Press.

Potter, Parker B. 1992. «Critical Archaeology: In the Ground and on the Street». Historical Archaeology 26 (3): 117-129. http://dx.doi.org/10.1007/BF03373547.

Preucel, Robert W. y Lynn Meskell. 2004. «Knowledges», en A Companion to Social Archaeology, Lynn Meskell y Robert W. Preucel, eds., pp. 3-22. Malden: Blackwell.

Renfrew, Colin. 2001. «From Social to CognitiveArchaeology:An Interview with Colin Renfrew». Journal of Social Archaeology 1 (1): 13-34. http://dx.doi.org/10.1177/146960530100100102 
Rodríguez Corral, Javier. 2006. «Textos y contextos de la materialidad e imaginación arqueológica». Galleacia 25: 305-331.

Shanks, Michael y Christopher Tilley. 1992. Re-constructing Archaeology. Theory and Practice. Londres y Nueva York: Routledge.

Smith, Monica L. 2007. «Inconspicuous Consumption: Non-display Goods and Identity Formation». Journal of Archaeological Method and Theory 14 (4): 412-438

Torres Caballero, José Vidal. 1994. «Reflexiones sobre la terminología lingüístico-gramatical». Cauce: Revista de filología y su didáctica 17: 83-106.

Trigger, Bruce G. 1992 (1989). Historia del pensamiento arqueológico. Barcelona: Editorial Crítica.

Vaquer, José María. 2012. «Apuntes para una semiótica de la materialidad». Comechingonia 16 (1): 13-29.

Weitz, Morris. 1977. The Opening Mind. A Philosophical Study of Humanistic Concepts. Londres: University of Chicago Press.

Wittgenstein, Ludwig. 2006 (1969). Sobre la certeza. Barcelona: Editorial Gedisa. 